\title{
ON THE COMPACTNESS OF THE STRUCTURE SPACE OF A RING
}

\author{
WUHAN LEE
}

1. Introduction. N. Jacobson [2, p. 204] has shown that a topology can be defined on the set $S(A)$ of primitive ideals of any nonradical ring $A$. With this topology, $S(A)$ is called the structure space of $A$. The topology is given by defining closure: If $T=\{P\}$ is a set of primitive ideals then the closure of $T, \mathrm{Cl} T$ is the set of primitive ideals which contain

$$
D T=\bigcap\{P \mid P \in T\} .
$$

It is well known that if $A$ has an identity element, then $S(A)$ is compact [2, p. 208]. Moreover, M. Schreiber [3] has observed that if every two-sided ideal of $A$ is finitely generated, then $S(A)$ is again compact. Further, R. L. Blair and L. C. Eggan [1] have obtained a result for a class of rings consisting of those $A$ such that

(C) no nonzero homomorphic image of $A$ is a radical ring

stating that the structure space of such a ring is compact if and only if $A$ is generated, as an ideal, by a finite number of elements.

The author found that for a certain class of rings, the modularity of the radical is both necessary and sufficient for the compactness of $S(A)$, and also that $S(A)$ is locally compact. For each $a \in A$, write (a) for the principal two-sided ideal generated by $a$, and let

$$
U_{a}=\{P \mid P \nsupseteq(a)\} \text {. }
$$

Then $\left\{U_{a}\right\}, a \in A$, is an open basis of the topology [3]. The author is interested in a ring $A$ such that

$$
\text { for every } a \in A, D U_{a} \text { is modular. }
$$

A two-sided ideal $P$ is modular in the sense that there exists a twosided identity modulo $P$.

2. Main results. Let $A$ be a ring with the property that $U_{a}$ is modular for every $a \in A$ (in this section).

THEOREM 1. The structure space of $A$ is compact if and only if the radical $R$ of $A$ is modular.

Proof. Suppose $S(A)$ is compact. Since $\left\{U_{x}\right\}, x \in A$, is an open

Received by the editors March 9, 1967. 
cover, there exists a subcover $\left\{U_{a}\right\}, a \in E$, where $E$ is a finite subset of $A$. Then

$$
\underset{a \in B}{D U} U_{a}=D S(A)=R
$$

since $\bigcup_{a \in E} U_{a}=S(A)$. But

Hence

$$
D U_{x \in A} U_{a} \supseteq \bigcap_{a \in E} D U_{a}
$$

$$
R \supseteq \bigcap_{\mathbf{a} \in \boldsymbol{B}} D U \text {. }
$$

By hypothesis, each $D U_{a}, a \in E$, is modular. Then it follows that the radical $R$ of $A$ is modular since an intersection of a finite number of modular two-sided ideals is modular.

Conversely, suppose $R$ is modular with an identity $e$ modulo $R$. Then $A / R$ is clearly a ring with an identity. Since $A / R$ has an identity, the structure space $S(A / R)$ is compact. Hence it follows that $S(A)$ is compact because $S(A)$ is homeomorphic to $S(A / R)$ by the corollary in $[2$, p. 205].

THEOREM 2. The structure space of $A$ is locally compact.

Proof. Let $P$ be a point of $S(A)$ and take $U_{a}$ as an open neighborhood of $P$. Since $D U_{a}$ is modular, $S\left(A / D U_{a}\right)$ is compact. Therefore its homeomorphic image $\mathrm{Cl} U_{a}$ is compact and thus $S(A)$ is locally compact.

3. Examples. A biregular ring $A$, in the sense that if $a \in A$ then there exists a central idempotent element $e$ such that $(a)=(e)$, satisfies the condition $\left(C^{\prime}\right)$, for it is easily seen that the element $e$ is an identity modulo every primitive ideal that does not contain $(a)$. But the ring of integers satisfies the condition $\left(C^{\prime}\right)$ while it fails to be a biregular ring.

It is further investigated that the condition $\left(C^{\prime}\right)$ does not imply the condition (C), and vice versa.

EXAMPLE 1. Let $B$ be a simple ring with an identity element and let $R$ be a nonzero radical ring, and let $A$ be the direct sum $B \oplus R$. Then $A$ has exactly one primitive ideal, namely, $R$. Thus, for $a \in A$, either

$$
D U_{a}=A \quad \text { (if } a \in R \text { ) or } \quad D U_{a}=R \quad \text { (if } a \notin R \text { ). }
$$

In either case, $D U_{a}$ is modular, so that $A$ satisfies $\left(C^{\prime}\right)$. However, $A / B \cong R$ is a radical ring, so $A$ does not satisfy (C).

ExAmple 2. Let $A$ be a simple ring without an identity element 
and not a radical ring. Clearly $A$ satisfies (C). The only primitive ideal is the zero ideal (0). If $a \in A$ with $a \neq 0$, then $D U_{a}=(0)$. Since $A$ has no identity element, $D U_{a}$ is not modular, so $A$ does not satisfy $\left(\mathrm{C}^{\prime}\right)$.

REMARKs. The author should like to express his thanks to the referee for his motivational suggestions.

\section{REFERENCES}

1. R. L. Blair and L. C. Eggan, On the compactness of the structure space of a ring, Proc. Amer. Math. Soc. 11 (1960), 876-879.

2. N. Jacobson, Structure of rings, Amer. Math. Soc. Colloq. Publ., Vol. 37, Amer. Math. Soc., Providence, R. I., 1956.

3. M. Schreiber, Compactness of the structure space of a ring, Proc. Amer. Math. Soc. 8 (1957), 684-685.

Seoul National University 